\title{
Plasmonic surface lattice resonances in arrays of metallic nanoparticle dimers
}

\author{
A. D. Humphrey and W. L. Barnes \\ School of Physics and Astronomy, University of Exeter, Stocker Road, Exeter, Devon, \\ EX4 4QL, United Kingdom \\ E-mail: A.D.Humphrey@exeter.ac.uk, W.L.Barnes@exeter.ac.uk
}

\begin{abstract}
We investigate the optical response of square arrays of metallic nanoparticles where each lattice site is occupied by two particles, a dimer. In particular we examine the surface lattice resonances arising in these structures when the inplane dipole moments associated with the plasmon modes of the nanoparticles couple together. The addition of a second particle to the basis leads to a more complex optical response, one that is anisotropic in the plane of the array. Extinction spectra are recorded at normal incidence for different orientations of the incident electric field. We compare our experimentally derived data with those from a coupled-dipole model. We show how the separation between the particles that comprise the dimer helps determine the overall response of the system.
\end{abstract}

Submitted to: J. Opt. 


\section{Introduction}

Light incident upon a metallic nanoparticle can drive the particle's conduction electrons into a collective oscillation [1]. This collective electron motion, which results in resonantly enhanced absorption and scattering of the incident light, is known as a localized surface plasmon resonance, or sometimes as a particle plasmon $[2,3]$. A change in the shape $[4,5]$, size $[6,7]$ or dielectric environment $[8,9]$ of the particle can alter the spectral position of the particle plasmon resonance. The resonant scattering property associated with the particle plasmon allows the plasmon mode of one particle to couple with another via their electromagnetic fields $[10,11,12]$.

When the separation is appropriate ( $\sim$ the single particle resonant wavelength) then, when both particles are illuminated by incident light, the field scattered by one particle may drive its neighbour constructively (destructively), i.e. the scattered field at the site of the neighbour has the same (opposite) phase as the incident field at the neighbour [12].

In an array of such particles collective resonances known as surface lattice resonances (SLRs) may occur, involving the constructive interference of scattered fields produced by all the particles that make up the array. Early works $[13,14,15,16,17$, 18, 19] focussed on particle plasmon resonances that had dipole moments in the plane of the array. SLRs based on out-of-plane dipole moments have also been investigated using oblique angle of incidence excitation $[19,20]$ and by employing a more complex particle shape [21].

SLRs have been investigated in a number of contexts including: sensing [22], strong coupling between SLRs and molecular transitions [23, 24] and lasing [25, 26]. The work we report here is part of an exploration into the SLRs supported by square arrays of metallic dimers, i.e. the basis has two metallic nanoparticles rather than the more usual one-particle basis. Dimer arrays have been investigated before, but predominantly for out-of-plane dipole moments [19, 27, 28], here our focus is on in-plane dipole moments. Whilst out-of-plane SLRs can be supported by arrays of plasmonic nanoparticles in an asymmetric environment (i.e. the media on either side of the particle array have different refractive indices), in-plane SLRs require a homogeneous environment [16]. Here we employ a homogeneous environment in our studies, and we look at how the optical response depends on the orientation of the incident electric field relative to the dimer axis. We also examine how the response depends on the inter-particle separation. Here we examine dimers in which the particles are identical. In a follow-up report [29] we will discuss asymmetric dimers, i.e. dimers in which the two particles are of different size.

In what follows we describe our results and discuss how they may be understood. We fabricated one- and two-particle basis square arrays of silver nanoparticles using electron-beam lithography (EBL) (details are given elsewhere [18]). We probed the optical response of our samples using normal-incidence extinction micro-spectroscopy and used our measurements to determine the extinction cross-section of the particles in 
the array. We compared the results of these experiments with a simple coupled-dipole model in which we treated each particle as a single polarizable unit. We found the model was sufficient to explain the main features of the recorded spectra, i.e. the spectral position, relative strength and width of the SLRs. Before describing our experimental results, we briefly outline the basis of the theoretical model.

We seek a method to describe the effect of the array structure on the polarizability of any given particle in the array. If we assume the array to be infinite (this approximation has been shown to be valid for arrays of the size we study here [17]) then we can make use of an analytic model. The advantage of an analytic model over numerical approaches, e.g. finite-element modelling, Mie theory etc., is that it provides a more intuitive approach that helps build physical understanding. Thus, whilst the analytic model we employ, known as the modified long-wavelength approximation (MLWA), has some shortcomings it is nonetheless a valuable tool [30].

For an infinite array of nanoparticles, the effective polarizability of one particle in the array, $\alpha_{\text {eff }}$, is related to the polarizability of the isolated particle, $\alpha$, by, [13, 31],

$$
\alpha_{\mathrm{eff}}=\frac{1}{1 / \alpha-\varepsilon_{0} S},
$$

where $\varepsilon_{0}$ is the permittivity of free space and the factor $S$, the lattice sum, takes account of the effect of all the scattered fields produced by all the other particles in the array. For a planar 2D array illuminated at normal incidence, $S$ is given by [32],

$$
S=\frac{1}{4 \pi \varepsilon_{0}} \sum_{j} \exp \left(i k r_{j}\right)\left[\frac{\left(1-i k r_{j}\right)\left(3 \cos ^{2} \theta_{j}-1\right)}{r_{j}^{3}}+\frac{k^{2} \sin ^{2} \theta_{j}}{r_{j}}\right],
$$

where $r_{j}$ is the distance from the central particle to all other particles $j$, and $\theta_{j}$ is the angle between $\vec{r}_{j}$ and the dipole moment of the central particle. The polarizability in the MLWA takes account of both radiation damping and retardation [33, 34] and is given by,

$$
\alpha=\frac{4 \pi \alpha_{\mathrm{qS}}}{4 \pi-\frac{2}{3} i k^{3} \alpha_{\mathrm{qS}}-\frac{k^{2}}{a} \alpha_{\mathrm{qS}}}
$$

where $k$ is the wavenumber in the medium in which the particles are embedded and $\alpha_{\mathrm{qs}}$ is the quasi-static polarizability, given by [35],

$$
\alpha_{\mathrm{qS}}=4 \pi a b c \frac{\varepsilon_{m}-\varepsilon_{s}}{3 \varepsilon_{s}+3 L\left(\varepsilon_{m}-\varepsilon_{s}\right)},
$$

where $a, b$ and $c$ are the semi-axes of the particle (an ellipsoid is the most general shape for which the electric field is uniform in the particle - a requirement of the quasi-static approximation [36]), $\varepsilon_{s}$ is the relative permittivity of the surrounding medium, $\varepsilon_{m}$ is the relative permittivity of the material from which the particle is made and $L$ is a geometrical factor that relates the shape of the particle to the internal electric field. In practice the array factor $S$ is calculated for finite-sized arrays $[17,16]$. One consequence of the finite size of the array is that the real and imaginary parts of the $S$-factor show 
rapid oscillations [37]. Therefore before using $S$, e.g. in (1), our $S$-factor data were smoothed using a cubic spline to remove these oscillations [38].

SLRs occur at the poles of (1), i.e. when the real part of $1 / \alpha$ equals $\varepsilon_{0} S$, provided the difference between their imaginary parts is small [21, 39, 40, 41]. For the calculations presented here arrays of $400 \times 400$ particles (one-particle basis) and $280 \times 280$ dimers (two-particle basis) were considered. Particles were approximated as oblate spheroids and given a polarizability calculated using the MLWA, (3). To calculate the optical response of the arrays, the effective polarizability (1) of the particle in the array is substituted into the expressions for the absorption and scattering cross-sections, given by [35], i.e.,

$$
\sigma_{\mathrm{abs}}=k \Im(\alpha)
$$

and,

$$
\sigma_{\mathrm{sca}}=\frac{k^{4}}{6 \pi}|\alpha|^{2}
$$

The extinction cross-section is then the sum of the absorption and scattering crosssections, i.e.,

$$
\sigma_{\text {ext }}=k \Im(\alpha)+\frac{k^{4}}{6 \pi}|\alpha|^{2}
$$

To facilitate comparison with the experimental data, all of the calculated extinction spectra discussed below were smoothed using a Gaussian [42] to replicate the 7 $\mathrm{nm}$ resolution of the spectrometer used in the experiments. Further, the measured transmittance data were converted to extinction cross-section per particle/dimer using,

$$
\sigma_{\text {ext }}=A(1-T)
$$

where $A$ is the area per particle/dimer within each array studied (the array footprint was larger (by about $60 \%$ ) than the illumination spot).

The work described below is based on arrays of circular silver discs (typically diameter $d \sim 85 \mathrm{~nm}$, height $h \sim 30 \mathrm{~nm}$ ). In figure 1 we show the (a) extinction cross-section (black solid line) and (b) phase of the polarizability (purple solid line) calculated for an isolated disc using (3) and (7); the permittivity for silver is taken from [43], the particles were assumed to be embedded in a medium of refractive index 1.515. Also shown in figure 1 are the spectral positions of the diffraction edges that correspond to the periods of the arrays studied. The periods (diffraction edges) are: 350 (530), 400 (606), 450 (682) and 500 (758) nm respectively.

\section{Square arrays with one-particle basis}

To provide a convenient context for discussing the results from arrays with a two-particle (dimer) basis, it is useful first to review the optical response of square arrays with a 


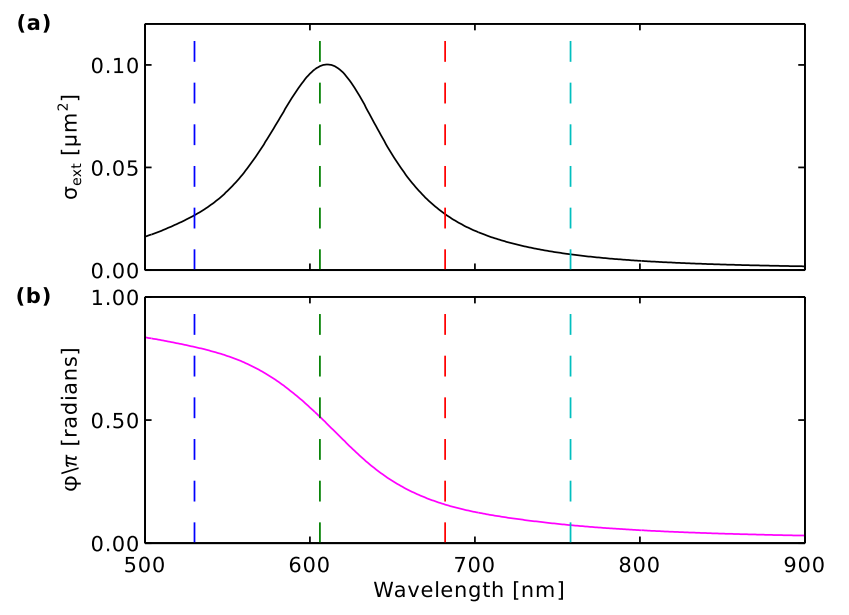

Figure 1. (a) extinction cross-section (black solid line) and (b) phase of polarizability (purple line) of a single silver disc $(d=85 \mathrm{~nm}, h=30 \mathrm{~nm})$ calculated using the modified long-wavelength approximation. The particle was embedded in a homogeneous medium with a refractive index $n=1.515$ with the incident electric field in the plane of the particle. Diffraction edges for the different array periods are illustrated by corresponding coloured dashed lines: 350 (blue), 400 (green), 450 (red) and $500 \mathrm{~nm}$ (cyan).

one-particle basis. Figure 2 shows scanning-electron micrographs (SEMs) of examples of (a) one- and (b) two-particle basis square arrays of silver discs $(d=85 \mathrm{~nm}$ and $h=30$ $\mathrm{nm}$ ) fabricated by EBL. In this particular case, both square arrays have a period of 400 nm.

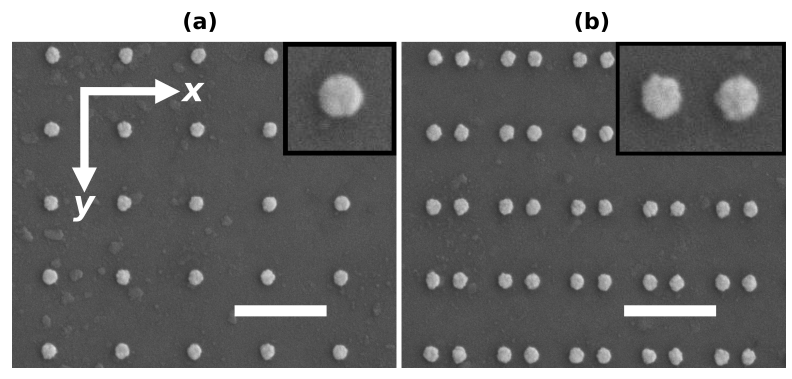

Figure 2. Scanning-electron micrographs (SEMs) of two different square arrays of silver discs $(d=85 \mathrm{~nm}$ and $h=30 \mathrm{~nm})$ : (a) a simple one-particle basis and (b) a two-particle (dimer) basis. The period of both arrays is $400 \mathrm{~nm}$. Scalebar=500 nm.

The measured extinction spectra per particle of arrays with period 350, 400, 450 and $500 \mathrm{~nm}$ for normally-incident light are displayed in figure 3 for two orthogonal orientations of the incident electric field: (a) parallel to the $x$-axis and (b) parallel to the $y$-axis of the array. For each different period of array the corresponding diffraction edge is illustrated by a dashed line of the same colour. In the measured extinction spectra, with the incident electric field parallel to the $x$-axis (see figure $3(\mathrm{a})$ ), a peak is seen in each spectrum (at 586, 620, 682 and $752 \mathrm{~nm}$ ) and is attributed to the SLR of the array. 
The SLRs occur spectrally closer to the diffraction edge as the period of the array is increased. This is because the phase of the particle polarizability at these longer wavelengths is smaller (see purple solid line in figure 1(b)) so that the electron motion more closely follows the applied electric field; the reduced phase difference results in the SLR occurring spectrally nearer the diffraction edge. Note that due to the symmetry of the particles and the symmetry of the array there is little difference between the measured extinction spectra of the two orthogonal directions of the incident electric field. We attribute the differences that remain to a residual lack of circular symmetry of the discs arising from imperfections in the fabrication process.

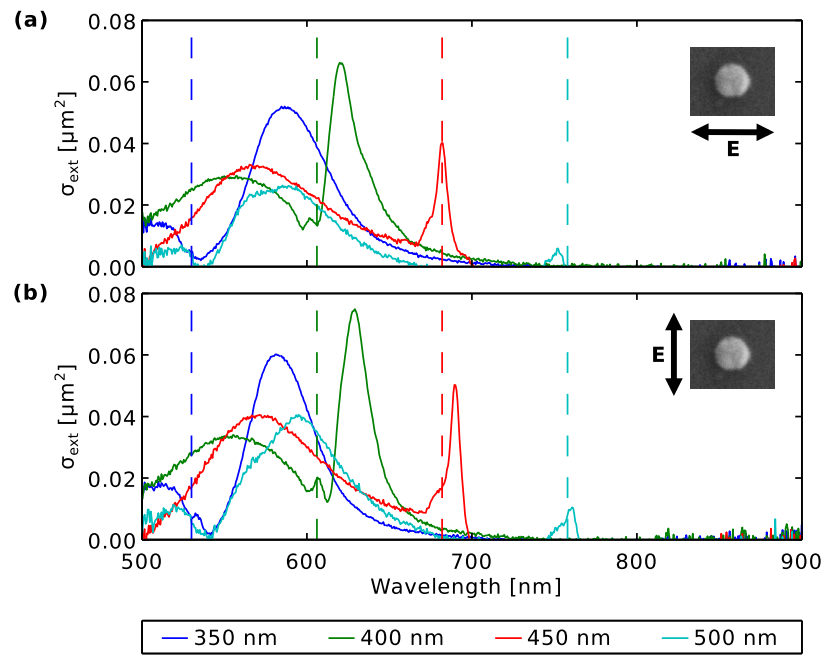

Figure 3. One-particle basis square arrays. Measured extinction cross-section per particle vs. wavelength for four different periods: 350, 400, 450 and $500 \mathrm{~nm}$. The diffraction edge associated with each different array period is illustrated by the corresponding coloured dashed line (530, 606, 683 and $758 \mathrm{~nm}$ ). The particles were silver discs, $d=85 \mathrm{~nm}$ and $h=30 \mathrm{~nm}$. The incident electric field was parallel to the $x$-axis of the array in (a) and parallel to the $y$-axis in (b). The environment of the particles was index-matched with the substrate using oil $(n=1.515)$. Colour legend displayed in bottom panel.

\section{Square arrays with two-particle (dimer) basis}

We now turn our attention to the SLRs supported by two-particle (dimer) basis square arrays and look at the dependence of their optical response on the orientation of the incident electric field. We investigated arrays with the same four values of the array period as for the one-particle basis arrays, and for the same particle size $(d=85 \mathrm{~nm}$ and $h=30 \mathrm{~nm}$ ). Figure 2(b) shows an SEM of one example of the dimer arrays. Figure 4 shows the $(\mathrm{a}, \mathrm{b})$ measured and $(\mathrm{c}, \mathrm{d})$ calculated extinction cross-section per dimer of the two-particle basis square arrays. The incident electric field was parallel to the dimer axis in $(a, c)$ and perpendicular in $(b, d)$. 
First let us consider the case when the incident electric field is parallel to the dimer axis. Comparing these data with those from the one-particle basis arrays (see figure 3 ), we see that the spectra are similar. The main difference, evident from the data, are that the SLRs in the two-particle basis arrays for $450 \mathrm{~nm}$ (red solid line) and $500 \mathrm{~nm}$ (cyan solid line) period arrays have an increased strength. The similar optical response between the one- and two-particle basis arrays occurs because particles in arrays where the period is on the order of the resonant wavelength couple most strongly to neighbours in the direction orthogonal to the applied electric field $[18,44]$. Since the separation between the particles in the $y$-direction (orthogonal to the applied electric field) of the two-particle basis array is the same as for the square array with a one-particle basis, a similar response is expected. The increase in strength of the SLR is attributed to there now being two particles per lattice site that contribute to the scattered fields. The proximity of the two particles in the basis probably accounts for the slight red-shift in the SLR [10, 45, 46].

Next let us consider the case when the incident electric field is perpendicular to the dimer axis. In the measured extinction cross-section per dimer spectra (see figure 4(b)), a single SLR at 566, 613, 682 and $754 \mathrm{~nm}$ is observed for square arrays with period 350, 400, 450 and $500 \mathrm{~nm}$. As noted above, inter-particle coupling takes place primarily in the direction orthogonal to the applied electric field. It might, thus, seem surprising that there is a strong SLR for this incident electric field orientation - the nearest particle is only $\lambda / 3$ away and should, therefore, dominate the interference condition (lattice sum). However, the data show that the SLRs for 350, 450 and $500 \mathrm{~nm}$ period square arrays with a two-particle basis appear similar in spectral shape and strength to the one-particle basis case (compare figures 3 and 4(b)). The largest difference in spectral shape between the one- and two-particle basis is for the $400 \mathrm{~nm}$ period arrays (compare green solid line in figures 3 and $4(\mathrm{~b})$ ). The shape of the extinction spectra for the $400 \mathrm{~nm}$ period square array also shows the greatest difference between the two incident electric field orientations for the two-particle basis (compare green solid line in figures 4(a) and 4(b)). Let us now compare the results just discussed with those obtained from the coupled-dipole model.

Let us again look at the case when the incident electric field is parallel to the dimer axis. Figure 4(c)) shows the result of the calculation whilst the relevant experimentallyderived spectra are in figure 4(a)). The position of the SLRs in the model (experiment) are: 637 (589), 661 (634), 704 (688) and 771 (756) $\mathrm{nm}$ for the 350, 400, 450 and 500 $\mathrm{nm}$ period arrays respectively. The largest difference between the experimental and modelled data is in the spectral positions of the SLRs for the $350 \mathrm{~nm}$ period array. This may be due to the MLWA not being so applicable at these shorter wavelengths; as the product $k a$ becomes larger, the higher powers of $k a$ that have been neglected in the MLWA may become more important [47]. Also the SLR for the $500 \mathrm{~nm}$ period array (compare cyan solid line in figures $4(\mathrm{c})$ and $4(\mathrm{a})$ ) is stronger in the model than in the experiment. Since this SLR is also the narrowest, it is possible that this difference is due to inhomogeneity of the particle sizes in the sample [48]. The significantly 


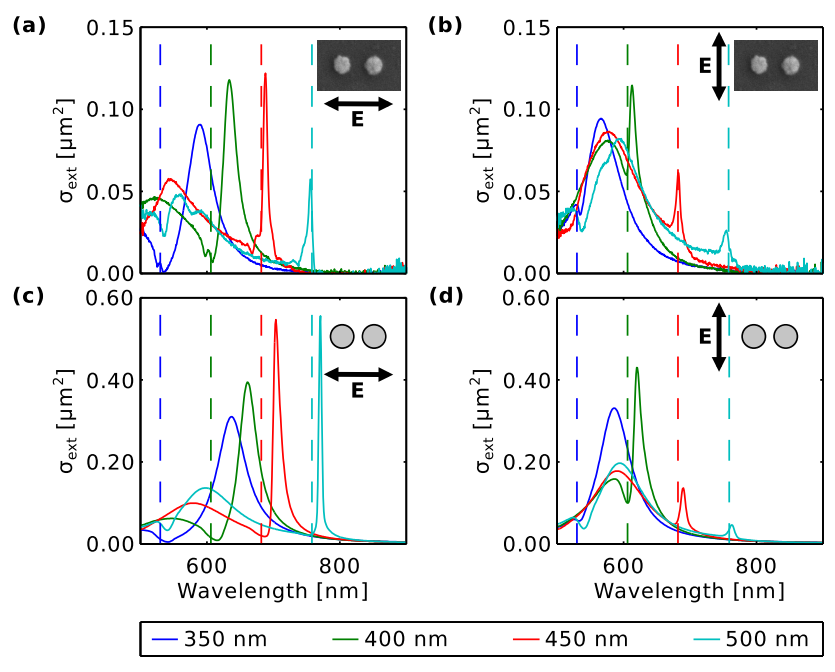

Figure 4. Two-particle basis (dimer) square arrays. Data for four different periods are shown: 350, 400, 450 and $500 \mathrm{~nm}$. The diffraction edge associated with each different array period is illustrated by the corresponding coloured dashed line $(530,606,683$ and $758 \mathrm{~nm})$. (a,b) measured and $(\mathrm{c}, \mathrm{d})$ calculated extinction cross-section per dimer $v s$. wavelength are shown, particles being silver discs $(d=85 \mathrm{~nm}$ and $h=30 \mathrm{~nm})$. The particle centre-to-centre separation in the basis was $150 \mathrm{~nm}$. The incident electric field was either (a.c) parallel to the dimer axis or (b,d) perpendicular. Colour legend displayed in bottom panel.

larger extinction cross-section per dimer as calculated in the model when compared to experiment is due to the polarizability in the MLWA overestimating the imaginary part of the single-particle polarizability; however, the MLWA does predict the right spectral shape, see [29].

When the incident electric field is perpendicular to the dimer axis, a comparison of the experimental data (see figure 4(b)) with the modelled data (see figure 4(d)) reveals generally good agreement although again the calculated extinction cross-sections are significantly greater than the measured ones.

At this point it is worth noting that in the modelling carried out above we considered all of the particles in the array as separate polarizable units. It is also possible to consider the particle dimer as one polarizable unit as, for example, considered by Pinchuk and Schatz [49]. We also undertook calculations (not shown) using the approach adopted by Pinchuk and Schatz but found significantly poorer correspondence with the results of our experiments than we have described above. In the model proposed by Pinchuk and Schatz retardation of the scattered light between the two particles in the dimer was ignored. As we discuss below, for the arrays considered here, such retardation can not be ignored. 


\section{Effect of particle separation within the dimer on the response of square array}

To probe the effect of particle separation in the dimer on the optical response of twoparticle basis square arrays, a number of samples were made that were nominally identical except for a difference in the inter-particle separation within the pair. More specifically, $500 \mathrm{~nm}$ period square arrays of dimers (particle size $d=115 \mathrm{~nm}$ and $h=30 \mathrm{~nm}$ ) with inter-particle separations of 160, 180, 200 and $220 \mathrm{~nm}$ in a $500 \mathrm{~nm}$ were fabricated and characterized. Figure 5 shows the (a,b) measured and (c,d) modelled extinction cross-sections.

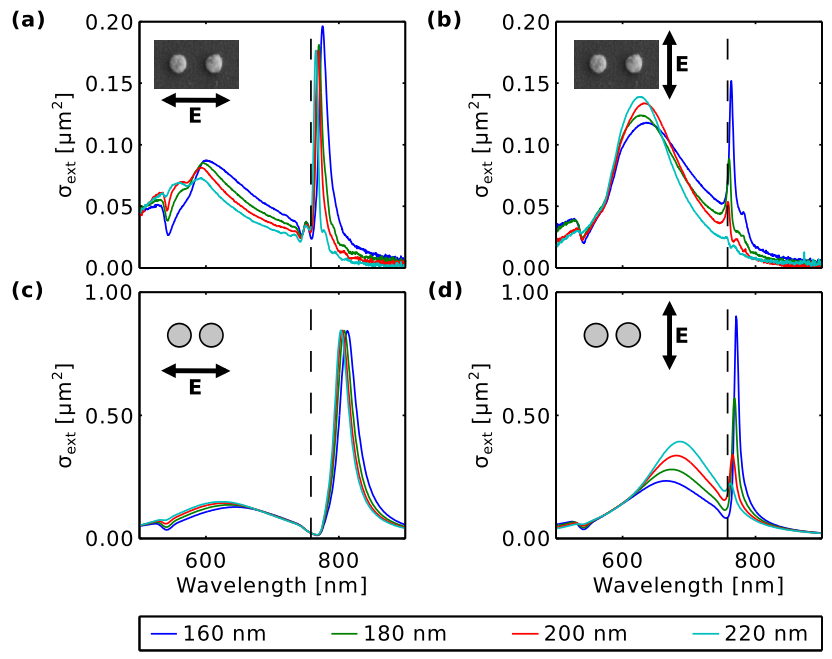

Figure 5. Square arrays with a two-particle basis. Data are shown for arrays of dimers of silver discs $(d=115 \mathrm{~nm}$ and $h=30 \mathrm{~nm})$ for four different dimer centreto-centre separations: 160, 180, 200 and $220 \mathrm{~nm}$. All arrays have a $500 \mathrm{~nm}$ period. $(\mathrm{a}, \mathrm{b})$ measured and $(\mathrm{c}, \mathrm{d})$ calculated extinction cross-section per dimer are shown. Data are shown for the incident electric field $(\mathrm{a}, \mathrm{c})$ parallel and $(\mathrm{b}, \mathrm{d})$ perpendicular to the dimer axis. The environment of the particles was homogeneous with $n=1.515$. The diffraction edge is illustrated with a black dashed line. Colour legend displayed in bottom panel.

What is striking about these data is the extent of the difference in the response for the two incident electric-field orientations. With the electric field parallel to the dimer axis, the response changes little with inter-particle separation within the dimer. Very different behaviour is seen when the electric field is perpendicular to the dimer axis; the SLR is almost completely lost for a separation of $220 \mathrm{~nm}$. This difference can again be attributed to the directional nature of the radiative coupling between particles. With the electric field perpendicular to the dimer axis, the scattered field experienced by one member of the dimer is dominated by that produced by the other member of the dimer. When the inter-particle separation is $\sim \lambda / 2$ this scattered field is out-of-phase with the driving electric field and a SLR is not produced. Conversely, for the parallel field orientation, the directional nature of the dipolar field is such that 
the resonant scattering that produces SLRs remains effective. We might also note that, for both field orientations, there are differences between experiment and theory regarding the width of the SLR. This might also be a result of the MLWA being used close to its limit of applicability. Further work would be needed to explore this in more detail.

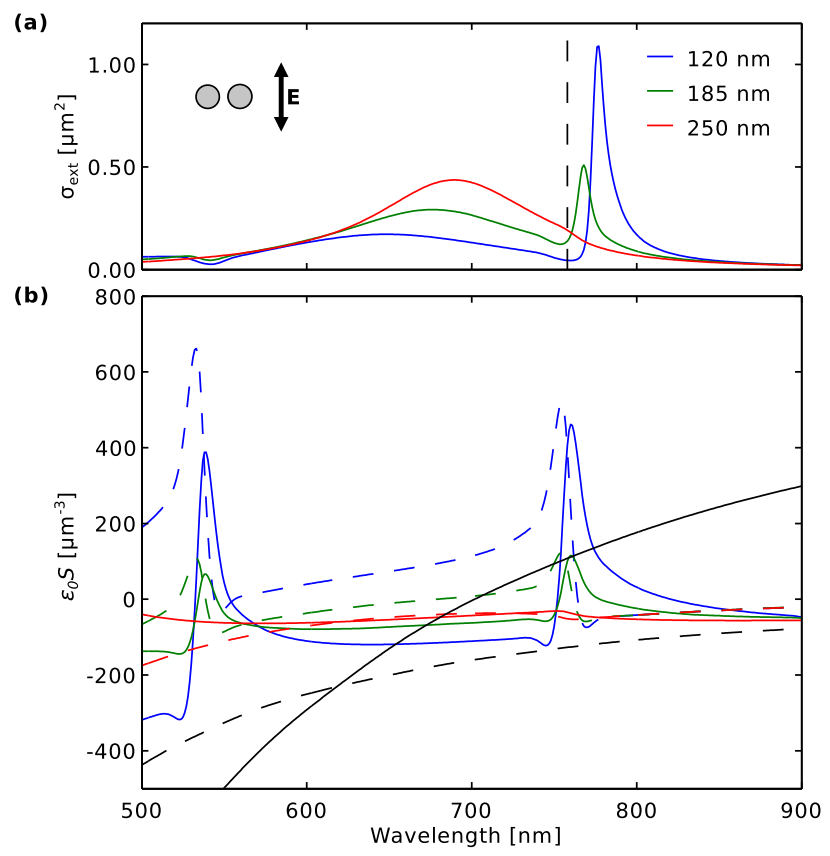

Figure 6. Coupled-dipole model calculated (a) extinction cross-section per dimer and (b) $\varepsilon_{0} S$ for $500 \mathrm{~nm}$ period square arrays with a two-particle basis. The real (black solid line) and imaginary part (black dashed line) of the inverse single particle polarizability of a disc $(d=115 \mathrm{~nm}$ and $h=30 \mathrm{~nm})$ was added to (b). Spectra are shown for three different centre-to-centre separations $(120,185$ and $250 \mathrm{~nm})$ with the incident electric field perpendicular to the dimer axis. The surrounding medium of the particles had $n=1.515$, and the diffraction edge is illustrated with a black dashed line.

Figure 6 shows the calculated (a) extinction cross-section per dimer and (b) $1 / \alpha$ and $\varepsilon_{0} S$, see (1), with the incident electric field perpendicular to the dimer axis for three inter-particle separations within the dimer. SLRs occur when the real parts of $1 / \alpha$ and $\varepsilon_{0} S$ intersect or have their closest approach (see (1)). The SLRs for inter-particle separations of $120 \mathrm{~nm}$ and $185 \mathrm{~nm}$ arrays are stronger because the real parts of $1 / \alpha$ and the real parts of $\varepsilon_{0} S$ intersect in the same spectral region as the diffraction edge, which does not happen with $250 \mathrm{~nm}$ separation array. The $120 \mathrm{~nm}$ inter-particle separation response is stronger than $185 \mathrm{~nm}$ one owing to the difference between the imaginary parts of $1 / \alpha$ and $\varepsilon_{0} S$ being smaller than for $185 \mathrm{~nm}$. The SLR with an inter-particle separation of $250 \mathrm{~nm}$ has nearly disappeared because the separation is approximately $\lambda / 2$ thus providing almost perfect destructive interference of the light scattered by the nanoparticles in the plane of the array. 


\section{Conclusion}

In summary, one- and two-particle basis square arrays of silver nanoparticles have been fabricated and characterized and their optical response (extinction cross-section) modelled using a simple coupled-dipole model. We showed that when the incident electric field is parallel to the dimer axis, the measured extinction cross-section vs. wavelength is similar to that exhibited by the equivalent one-particle basis square array. The perpendicular response of two-particle basis arrays is different from the equivalent one-particle basis arrays for certain periods of square array (i.e. $400 \mathrm{~nm}$ in this instance). The particles couple together in the direction that is orthogonal to the applied electric field, and for the perpendicular polarization, the nearest particle in this orthogonal direction is significantly closer than the next lattice site. Two-particle basis square arrays can not be modelled as a single polarizable unit because retardation of the electric field between the particles in the dimer can not be ignored. The optical response of the two-particle basis square arrays were discussed in the context of the scattered fields produced by particles in an array. The centre-to-centre separation of the particles in the dimer is important, and when this is approximately $\lambda / 2$, the SLR vanishes. Although a symmetric two-particle basis can support an anti-symmetric plasmon mode, we did

not observe it here. This is expected since this mode can not be coupled to at normal incidence because it has a zero net dipole moment, i.e. it does not radiate. However, it might perhaps be important in applications of dimer arrays to lasing structures [50, 51].

As others have shown, such modes can be excited by light, either by using nonnormal incidence [20], or through the use of complex particle morphology [21]. An alternative strategy, that of introducing an asymmetry into the dimer will be the subject of a follow-on report [29].

\section{Acknowledgements}

The support of The Leverhulme Trust and of the EPSRC (EP/K041150/1) is gratefully acknowledged. The authors would also like to thank Dr Nina Meinzer for many valuable discussions.

\section{References}

[1] Meinzer N, Barnes W L and Hooper I R 2014 Nature Photonics 8889

[2] Kreibig U and Vollmer M 2010 Optical Properties of Metal Clusters (Springer-Verlag Berlin)

[3] Hutter E and Fendler J H 2004 Advanced Materials 161685

[4] Mock J J, Barbic M, Smith D R, Schultz D A and Schultz S 2002 Journal of Chemical Physics 1166755

[5] Murray W A and Barnes W L 2007 Advanced Materials 193771

[6] Sönnichsen C, Franzl T, Wilk T, von Plessen G, Feldmann J, Wilson O and Mulvaney P 2002 Physical Review Letters $\mathbf{8 8} 077402$

[7] Anker J N, Hall W P, Lyandres O, Shah N C, Zhao J and Van Duyne R P 2008 Nature Materials 7442 
Plasmonic surface lattice resonances in arrays of metallic nanoparticle dimers

[8] Mock J J, Smith D R and Schultz S 2003 Nano Letters 3485

[9] Murray W A, Auguié B and Barnes W L 2009 Journal of Physical Chemistry C 1135120

[10] Rechberger W, Hohenau a, Leitner a, Krenn J R, Lamprecht B and Aussenegg F R 2003 Optics Communications 220137

[11] Nordlander P, Oubre C, Prodan E, Li K and Stockman M I 2004 Nano Letters 4899

[12] Dahmen C, Schmidt B and von Plessen G 2007 Nano Letters 7318

[13] Zou S, Janel N and Schatz G C 2004 Journal of Chemical Physics 12010871

[14] Markel V A 2005 Journal of Physics B 38 L115

[15] Chu Y, Schonbrun E, Yang T and Crozier K B 2008 Applied Physics Letters 93181108

[16] Auguié B and Barnes W L 2008 Physical Review Letters 101143902

[17] Rodriguez S R K, Schaafsma M C, Berrier A and Gómez Rivas J 2012 Physica B 407 4081-4085

[18] Humphrey A D and Barnes W L 2014 Physical Review B 90075404

[19] Kravets V G, Schedin F and Grigorenko A N 2008 Physical Review Letters 101087403

[20] Zhou W and Odom T W 2011 Nature Nanotechnology 6423

[21] Thackray B D, Kravets V G, Schedin F, Auton G, Thomas P A and Grigorenko A N 2014 ACS Photonics 11116

[22] Ng B, Hanham S M, Giannini V, Chen Z C, Tang M, Liew Y F, Klein N, Hong M H and Maier S A 2011 Optics Express 1914653

[23] Väkeväinen A I, Moerland R J, Rekola H T, Eskelinen A, Martikainen J P, Kim D H and Törmä P 2014 Nano Letters 141721

[24] Rodriguez S R K and Gómez Rivas J 2013 Optics Express 2127411

[25] Zhou W, Dridi M, Suh J Y, Kim C H, Co D T, Wasielewski M R, Schatz G C and Odom T W 2013 Nature Nanotechnology 8506

[26] Dridi M and Schatz G C 2015 Journal of the Optical Society of America B 32818

[27] Kravets V G, Schedin F, Taylor S, Viita D and Grigorenko A N 2010 Optics Express 189780

[28] Kravets V G, Schedin F, Pisano G, Thackray B, Thomas P A and Grigorenko A N 2014 Physical Review B 90125445

[29] Humphrey A D, Meinzer N and Barnes W L Submitted to ACS Photonics

[30] Moroz A 2009 Journal of the Optical Society of America B 26517

[31] García de Abajo F J 2007 Reviews of Modern Physics 791267

[32] DeJarnette D, Jang G G, Blake P and Roper D K 2014 Journal of Optics 16105006

[33] Wokaun A, Gordon J P and Liao P F 1982 Physical Review Letters 48957

[34] Meier M and Wokaun A 1983 Optics Letters 8581

[35] Bohren C F and Huffman D R 2004 Absorption 83 Scattering of Light by Small Particles (Wiley$\mathrm{VCH})$

[36] Kang H and Milton G W 2008 Archives of Rational Mechanical Analysis 18893

[37] Auguié B 2009 Optical properties of gold nanostructures Ph.D. thesis University of Exeter

[38] Smoothing was performed using the MATLAB function "csaps" with a smoothing parameter of 0.01

[39] Evlyukhin A B, Reinhardt C, Zywietz U and Chichkov B N 2012 Physical Review B 85245411

[40] Teperik T V and Degiron A 2012 Physical Review B 86245425

[41] Špačková B and Homola J 2013 Optics Express 2127490

[42] The MATLAB "filter" function was used with 7-points with $a=1$ and $b=$ $[0.054 ; 0.123 ; 0.203 ; 0.240 ; 0.203 ; 0.123 ; 0.054]$, where $a$ and $b$ are weighting coefficients

[43] Lynch D W and Hunter W R 1985 Handbook of Optical Constants of Solids (Academic, New York)

[44] Pinchuk A O and Schatz G C 2008 Materials Science and Engineering B 149251

[45] Gunnarsson L, Rindzevicius T, Prikulis J, Kasemo B, Käll M, Zou S and Schatz G C 2005 Journal of Physical Chemistry B 1091079

[46] Jain P K, Huang W and El-Sayed M A 2007 Nano Letters 72080

[47] Chung H Y, Leung P T and Tsai D P 2012 Plasmonics 713

[48] Auguié B and Barnes W L 2009 Optics Letters 34401 
[49] Pinchuk A and Schatz G 2005 Nanotechnology 162209

[50] Schokker A H and Koenderink A F 2014 Physical Review B 90155452

[51] Schokker A H and Koenderink A F 2015 ACS Photonics 21289 